\title{
Calibration of a silver thin film gauge for short duration convective step heat load
}

\author{
SHRUTIDHARA SARMA, NIRANJAN SAHOO* and AYNUR UNAL \\ Department of Mechanical Engineering, Indian Institute of Technology Guwahati, Guwahati 781 039, India \\ e-mail: shock@iitg.ernet.in
}

MS received 4 August 2015; revised 28 November 2015; accepted 9 January 2016

\begin{abstract}
Thin film gauges (TFGs) are a promising candidate for measuring transient heat fluxes in the applications involving very short duration of heating environment. They are basically resistance temperature detectors (RTDs) having the capability of responding in the range of few microseconds. In the present study, a silver thin film gauge (STFG) is fabricated and calibrated in-house with a view to assess the performance of STFGs in dynamic environment. Convective heat load is supplied by a hot-air gun where the heated air jet strikes the gauge and its response is obtained through voltage signal. Subsequently, the surface heat fluxes are estimated by using one dimensional heat conduction modeling. The similar experimental environment is studied to obtain the flow behavior of hot-air jet emanating into atmosphere by using numerical simulations. The selfsimilar velocities are plotted as well as the interference of outer domain into the experiment parameters has been studied. Ultimately, the surface heat fluxes obtained from various methods are compared to analyse the performance of this hand-made STFG. This study reveals the ability of STFGs to be used in practical short duration transient situations.
\end{abstract}

Keywords. Thin film gauge; temperature coefficient of resistance; step heat load; surface heat flux.

\section{Introduction}

Heat transfer measurement in short duration time scale is one of the difficult aspects due to the need for fast response temperature sensors. Many impulse aerodynamic test facilities do require measurements, where time scales can go up to even milliseconds or less. It calls for the need of fast response sensors for which the response time is fast enough to trace the temperature variations [1-6]. Although numerous sensors have been developed through decades, yet there are only few specific categories of thermal sensors, suitable for applications of highly transient environment. Thin film temperature gauge (TFG) is an efficient candidate in these applications since they can respond in microsecond durations due to their extremely small thickness $(\sim \mu \mathrm{m}$ or $\mathrm{nm})$. They are resistance temperature detectors (RTDs) consisting of thin metallic films deposited on the surface of insulating substrates [7, 8]. The resistance of the gauge is formed by depositing high conducting materials like platinum, nickel and silver (ink/paste/powder/solid form) on the insulating ceramic substrates (such as Pyrex/Macor). When the TFGs are exposed to transient environments of rapidly changing temperature, the resistance of the gauge changes which can be detected by voltage variation provided the temperature coefficient of

*For correspondence resistance (TCR) of the gauge is known as a priori. The measured time-dependent surface temperature of the gauge with the known thermal properties of substrate material allows the estimation of transient surface heat with appropriate heat conduction modeling [9-11]. These sensors have the capability of measuring any kind of heat input and therefore have been successfully implemented in aerospace applications for capturing transient surface temperature changes in a supersonic flight [12], surface temperature determination over aerodynamic bodies in short duration impulse facilities [13]. Their applications have been further extended to in trace the pulsating variation of surface temperature and heat flux in internal combustion engines [14] and real-time measurement of electrolyte temperature detector in a polymer electrolyte fuel cell too [15].

In thin film based transient temperature/heat flux measurements in short duration time scales, the convective heat loads are the dominant mode of heat transfer. There are potential research areas in which the transient measurements with TFGs can be extended to other modes of heat transfer (i.e., conduction and radiation). At any case, the calibration methodologies with all basic modes of heat transfer are highly essential because they represent the uncertainties in measurement of temperature and subsequent determination of surface heat fluxes in actual applications of these sensors. The radiation based dynamic calibration techniques for such heat flux micro-sensors have 
been reported using heat lamp [16] and pulse laser source [8]. In pure conduction mode of heat transfer experiments, the temperature history and subsequent predictions of surface heat fluxes have been reported for hand-made platinum TFGs [17]. In order to justify the effectiveness of TFGs in applications where the basic mode of heat transfer is 'convection', it is essential to calibrate such sensors in similar environments. The objective of the study is to devise a calibration method for TFG with respect to convective mode of heating for short duration transient heating environment. Thus, the implementation calibration method of TFG for convection based heat transfer and development of an experimental set-up are two important expected outcomes of this study. In this backdrop, simple laboratory experiments are designed in which a TFG is exposed with heated air flow generated by a "hot-air gun" of known wattage. The hand-made silver thin film gauge (STFG) of adequate resistance is prepared with 'pyrex' as substrate material. The experiments are carried out by exposing the silver TFG to various known step heat load of known input wattage, for the duration of $100 \mathrm{~ms}$. Then, the voltage signals are recorded due to change in temperature of air flow past the TFG. The one-dimensional heat conduction modeling is used to recover the heating loads for the transient temperature data and then compared with the known input heating loads. The numerical simulation (ANSYS-Fluent v. 14.5) is performed in the similar experimental environmental conditions, for same heating loads to obtain the expected convective heat load. The results of experiments and numerical simulation are compared for evaluating the performance of this STFG. Details of the experimental set-up, TCR estimation, experimental procedure and data reduction are discussed in the following sections.

\section{Fabrication and static calibration of thin film gauge}

A typical thin film gauge is comprised of an insulator as a substrate material painted or sputtered with a higher conducting thin film (platinum/nickel) as the sensing surface. In this particular application, STFGs are prepared with pyrex substrate having $6 \mathrm{~mm}$ diameter and $10 \mathrm{~mm}$ long. The thermal properties of the gauge and its substrate materials are given in table 1 [18]. While preparing the gauge, the surface of the substrate is smoothened with sand papers of various grain sizes (initially with 200 and later

Table 1. Thermal properties of TFG materials [18].

\begin{tabular}{lccc}
\hline Material & $\rho\left(\mathrm{kg} / \mathrm{m}^{3}\right)$ & $c_{p}(\mathrm{~J} / \mathrm{kg} \mathrm{K})$ & $k(\mathrm{~W} / \mathrm{m} \mathrm{K})$ \\
\hline Pyrex & 2220 & 775 & 1.3 \\
Silver & 10,490 & 233 & 429 \\
\hline
\end{tabular}

with 1000 grain size) and washed properly followed by adequate heating in an oven to make it moisture free. Then, thin films of silver paste are painted on the cleaned pyrex substrate with additional silver stripes on each side of the gauges to ease lead wire connections. Subsequently, the gauges are baked in oven by gradual heating up to a desired temperature $\left(350^{\circ} \mathrm{C}\right)$ followed by cooling to room temperature. The process of coating is repeated till the adequate resistance across the thin film (10-100 $\Omega$ ) is obtained. Since the TFGs are powered by a constant current source, these typical values of resistance help in measuring the signal in a suitable voltage range. The resistance of the films is measured with a standard four-probe resistivity measurement. The resultant resistance of the gauge depends on various factors like film thickness, material quality, and effective length of the film [18]. Electrical connections are made on the additional silver stripes by soldering lead wires on them and these lead wire connections are wrapped with 'Teflon' to secure them. The final resistance is measured with a multimeter at the end of the connecting wires. The typical STFG fabricated in-house with above procedure is shown in figure 1(a) and its resistance is found to be $16 \Omega$.

Basically, the TFGs are the temperature detectors for which the resistance increases with temperature rise in the flow environment. This increase in resistance can be interpreted as rise in voltage, if a constant current is applied across the gauge. The current is used to activate the sensor, as it is a passive device. Therefore, a voltage-temperature relation can be made through a parameter "TCR" for a particular TFG. Normally, the sensing element is chosen as pure metal, for which a linear relationship can be obtained between voltage and temperature. The thermal properties of sensing material and the resistance of the thin film are the important parameters which affect the TCR of the thin film sensor. Mathematically, the linear relationship for resistance $(R)$ at any arbitrary temperature $(T)$ can be represented as follows [19]:

$$
R(T)=R_{0}\left[1+\alpha\left(T-T_{0}\right)\right]
$$

Here, $R_{0}$ is the initial resistance of the gauge at temperature $T_{0}$ and $\alpha$ is the TCR of the gauge. Since, TFGs need to be activated using a constant current source, the resistance can be converted to voltage form by using Ohm's law and $\alpha$ can be represented by following expression:

$$
\alpha=\frac{1}{V_{0}}\left(\frac{V-V_{0}}{T-T_{0}}\right)=\frac{1}{V_{0}}\left(\frac{\Delta V}{\Delta T}\right) .
$$

The voltage parameters $\left(V_{0}\right.$ and $\left.V\right)$ represent the corresponding voltage values at their respective temperatures $\left(T_{0}\right.$ and $\left.T\right)$. It may be noted that the initial voltage is obtained for a known resistance TFG by supplying a suitable current across the TFG. While choosing the initial current, one has to careful about the fact that a measurable voltage output is obtained without the self-heating of the gauge. 


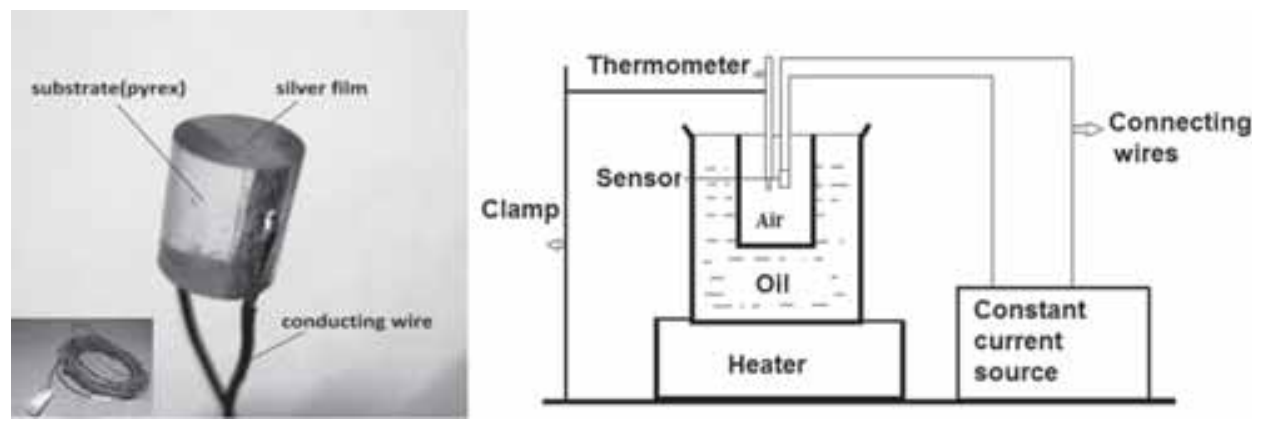

Figure 1. (a) Photograph of a silver TFG thin film gauge fabricated in the laboratory; (b) schematic diagram of oil-bath calibration experiment.

The standard "oil-bath calibration procedure" is followed for determination of TCR of the STFG [20]. For this purpose, an empty beaker containing the gauge is kept inside a silicon oil-bath and heat is supplied to the bath so that the TFG experiences temperature change through the hot air inside the empty beaker (figure $1 \mathrm{~b}$ ). Side by side, a thermometer (Fluke Hart Scientific 1523 thermometers) mounted in the beaker is used to monitor the temperature of the hot air. The TFG and the thermometer are kept as close as possible and the top of the empty beaker is covered with aluminum foil in order to avoid any thermal gradient. The TFG is energized by a constant current of $5 \mathrm{~mA}$ using a source meter (Series 2400-903-01, KEITHLEY, Cleveland, Ohio, USA). This value of current is sufficient to obtain a measurable output by using the same source meter. Considering the fact ohmic self-heating of the gauge, the further increase in current is avoided [18].

In the environment of oil-bath, the experiments are performed and the readings from TFG are recorded in the same source meter. The calibration curve for the gauge is shown (figure 2), in which the voltage values are plotted against temperature readings at an interval of $5^{\circ} \mathrm{C}$, during heating $\left(30^{\circ} \mathrm{C}\right.$ to $\left.70^{\circ} \mathrm{C}\right)$ and subsequent cooling $\left(70^{\circ}\right.$ to $\left.30^{\circ} \mathrm{C}\right)$ process. In order to check the repeatability of data, three sets of reading are taken in the heating and cooling process (figure $2 \mathrm{a}$ ). Then, the average value of voltage at each temperature is calculated and plotted in figure 2(b). Ideally, a thin film sensor should follow the same resistance path with increment and decrement of temperature. However, during the process of heating and cooling, the temperature of air increases initially followed by a decrease to original value. But, the change in voltage inside the material does not follow the same path because the internal field 'lags' behind the external field. This behavior results in loss of energy, commonly referred as "hysteresis loss" due to repeated heating and cooling. A linear trend is observed in the heating and cooling curve and the average value of slope of this curve is found to be $0.1548 \mathrm{mV} /{ }^{\circ} \mathrm{C}$ and

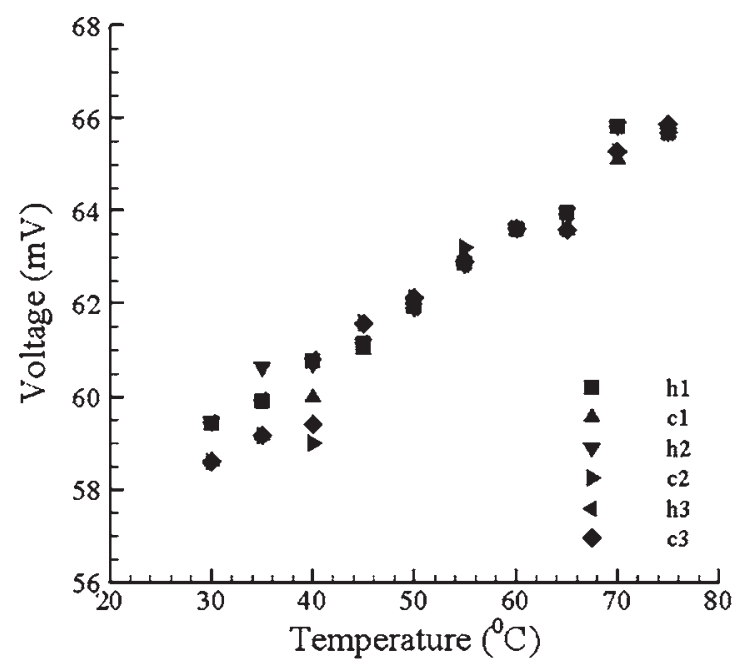

(a)

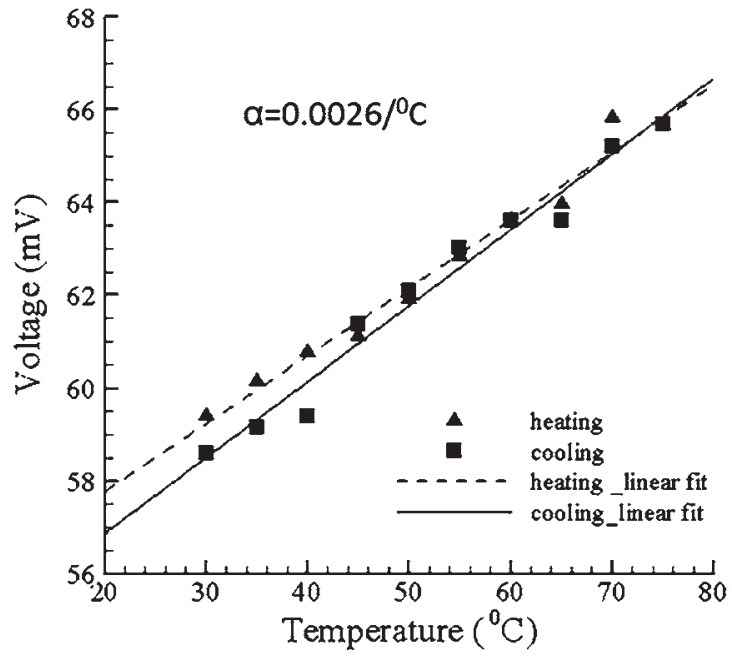

(b)

Figure 2. Static calibration experiment: (a) voltage-temperature data during heating and cooling process; (b) determination of TCR. 
essentially the TCR of the STFG is calculated as $0.0026 /{ }^{\circ} \mathrm{C}$ (Eq. 2). It may be noted that this value of TCR is comparable to that of platinum TFG $\left(0.0021 /{ }^{\circ} \mathrm{C}\right)$ as reported in the literature [8]. However, during short duration experiments, it is expected that a platinum TFG will respond faster as compared to a silver TFG. It mainly depends on the factors like film thickness, material property and purity of the materials. At any case, the determination of TCR essentially relates the voltage change corresponding to the temperature change. The co-efficient of accuracy during heating and cooling curves is 0.9787 and 0.9726 , respectively. It shows that the resistance of the gauge follows temperature almost linearly during heating and cooling process. Thus, the determination of TCR for a TFG enables the user for inferring temperature data from the voltage readings.

\section{Dynamic calibration study}

In most of the convective based experiments, the heat loads are typically step/impulsive in nature prevailing for a very short duration. Since, the TFGs have high response time, they can be used for capturing short duration transient temperatures for unknown convective heat loads. In order to resemble the similar nature of heat load, a simple laboratory experiment is designed for calibrating this STFG for known heat load. Then, the transient temperature response is recorded and subsequently used surface heat flux calculations with one-dimensional heat conduction modeling. Further, the numerical simulation is carried out to determine the heat flux by imposing boundary conditions with prevailing experimental parameters. This dynamic calibration essentially compares the recovery of the surface heat flux obtained through the hand-made STFG and heat flux predictions with various methods. The details of experimental setup, computation methodology and numerical simulations are discussed in this section.

\subsection{Experiments with TFG for convective heat load}

One of the important objectives of dynamic calibration is to expose the TFG in a heating environment of known heat load and subsequently acquiring its temperature response. For this purpose, the TFG is subjected to a step input heat load from a 'hot-air gun' of known wattage (figure 3 ). This instrument (Model: TGF 1500W) has a heater embedded in it that supplies hot-air jet to the atmosphere while operating in two modes (low and high). Based on manufacture's specification, it is supposed to generate a power of $1000 \mathrm{~W}$ in 'low mode' while the rated power in 'high mode' is 1500 $\mathrm{W}$. The gun has a nozzle of $20 \mathrm{~mm}$ diameter and generates hot air at a constant flow rate. The TFG is fixed on a plate at a distance of $10 \mathrm{~mm}$ from the exit of the nozzle with its

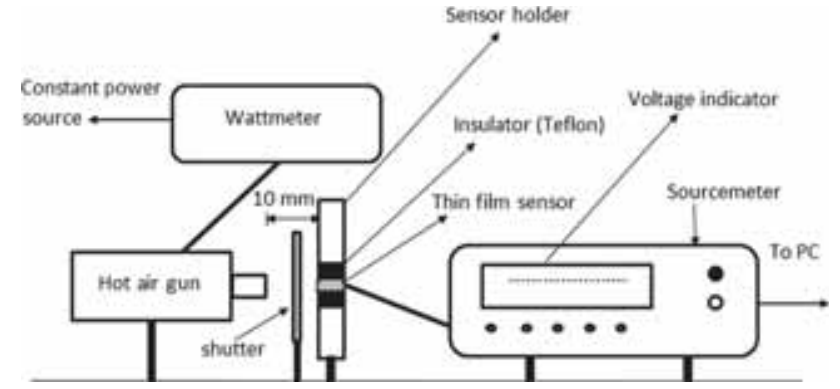

Figure 3. Schematic diagram of hot-air gun calibration experimental setup.

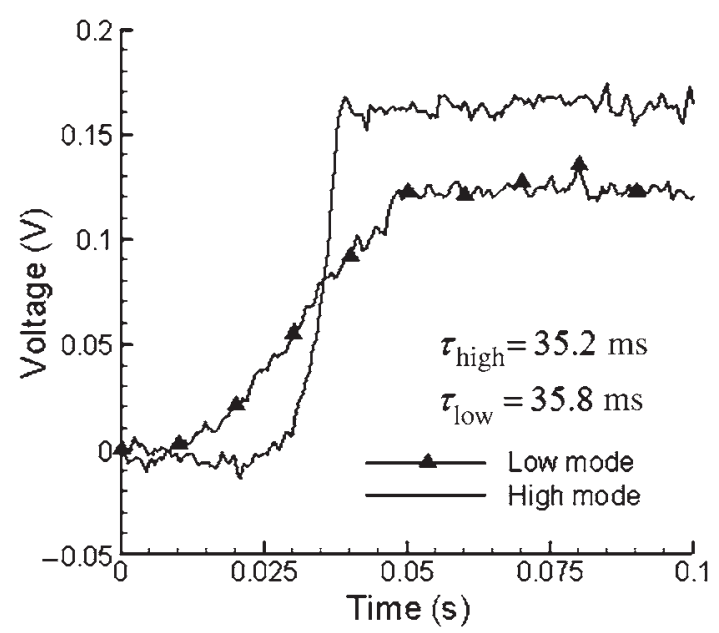

Figure 4. A typical voltage-time history from silver TFG during hot-air gun calibration experiment.

axis, aligned to the axis of the hot-air gun (figure 3). A shutter is placed in front of the TFG that remains closed unless full flow is achieved by the gun. Once the gun has achieved full flow (nearly after $10 \mathrm{~s}$ ) of starting it, the shutter is immediately opened so that the hot air from the gun can pass over the TFG surface as a jet. Subsequently, the voltage data due to the temperature change on the surface of TFG is captured for $100 \mathrm{~ms}$ duration (figure 4). After the initial unsteadiness, the steady state voltage variation is seen for about $50 \mathrm{~ms}$. Thus, the heating load on the TFG can be interpreted as a 'convective step heat load' prevailing for a duration of $50 \mathrm{~ms}$. Prior to the experiment, the rated power of hot-air gun is measured in the laboratory by using the 'wattmeter' and its efficiency is found to be $90 \%$ leading to the powers of $750 \mathrm{~W}$ and $1350 \mathrm{~W}$ in low and high mode, respectively. Based on these values, the heat fluxes $\left(\dot{q}_{i n}\right)$ are calculated based on area calculated with the nozzle diameter of the hot-air gun (table 2). The same source meter is integral part of TFG instrumentation that supplies constant current $(5 \mathrm{~mA})$ as well as records voltage readings at an interval of $0.4 \mathrm{~ms}$. Then, the voltage data is transferred through a terminal emulator (TeraTerm) and connecting the source meter to the computer with serial 
Table 2. Convective heating experiments and heat fluxes estimation.

\begin{tabular}{lccccc}
\hline Convective & $\begin{array}{c}\dot{q}_{\text {in }} \\
\text { Time } \\
\text { heating } \\
\text { gun mode }\end{array}$ & $\begin{array}{c}\dot{q}_{\text {exp }} \\
\left(\mathrm{W} / \mathrm{cm}^{2}\right)\end{array}$ & $\left(\mathrm{W} / \mathrm{cm}^{2}\right)$ & $\begin{array}{c}\dot{q}_{n} \\
\left(\mathrm{~W} / \mathrm{cm}^{2}\right)\end{array}$ & $\begin{array}{c}\text { Deviation }(\%) \\
\left(\frac{\dot{q}_{\text {in }}-\dot{q}_{\text {exp }}}{\dot{q}_{\text {in }}}\right) \times 100\end{array}$ \\
\hline Low & & & & 32 & $\begin{array}{c}\text { Deviation }(\%) \\
\left(\frac{\dot{q}_{n}-\dot{q}_{\text {exp }}}{\dot{q}_{n}}\right) \times 100\end{array}$ \\
High & 35.8 & 287 & 196 & 278 & 4 \\
\hline
\end{tabular}

communicator (RS-232). With several repeated trials of experiments, the typical voltage signals captured from TFG are shown in figure 4. It is seen that the gauge responds immediately as the hot-air jet passes over it and the 'time constant $(\tau)^{\prime}$ 'for both modes of operation are given in table 2. Experimentally, it is calculated as the time taken by the system to reach $63.2 \%$ of the full scale average [21].

The analytical method for the time constant of a sensor is given by the following expression:

$$
\tau=\frac{\rho C_{v} \forall}{h A},
$$

where $\rho$ is density, $C_{v}$ is thermal capacity, $\forall$ is volume, $A$ is surface area of the sensor and $h$ is the convective heat transfer co-efficient. Generally, the "time constant" is measured by suddenly exposing the sensor to a step change in temperature and the response of the sensor to step input is recorded and time-temperature data during the step change only is analyzed to calculate time constant. With respect to this experiment, the known convective loads are applied for smaller time scales $(0.1 \mathrm{~s})$ through hot-air gun and the sudden change in temperature is monitored. Then the convective heat transfer coefficient is calculated.

$$
h=\frac{q^{\prime \prime}}{\Delta T} .
$$

With the known input power and temperature difference in both the modes, the heat transfer co-efficient (using Eq. 4) are calculated as $9051 \mathrm{~W} / \mathrm{m}^{2} \mathrm{~K}$ and $8686 \mathrm{~W} / \mathrm{m}^{2} \mathrm{~K}$ for high and low modes, respectively. Considering film thickness as $120 \mu \mathrm{m}$, the time constants for low and high modes are calculated as $33.8 \mathrm{~ms}$ and $32.4 \mathrm{~ms}$, respectively (using Eq. 3). Experimentally (table 2; figure 4), the values are calculated as $35.8 \mathrm{~ms}$ and $35.2 \mathrm{~ms}$ for low and high modes, respectively, which are quite close to analytical values.

The surface heating rates from transient temperatures in short duration time scale can be recovered by using appropriate one-dimensional heat conduction modeling $[4,11]$. Since the thermal penetration distance during experimental run times is small compared to the linear dimension of the gauge, the system can be modeled by considering unsteady, linear conduction of heat in a onedimensional semi-infinite solid [11]. When substrate thermal properties are treated as constant, the heat flux $\left(\dot{q}_{\exp }\right)$, passing through surface is calculated from transient temperature $\left(T_{S}\right)$ by using Duhamel's superposition integral as given below;

$$
\dot{q}_{\exp }=\sqrt{\frac{\rho_{s} c_{s} k_{s}}{\pi}} \int_{0}^{t}\left(\frac{\mathrm{d} T_{s}}{\mathrm{~d} t}\right) \frac{1}{\sqrt{t-\tau}} \mathrm{d} \tau,
$$

where $\rho_{s}, c_{s}$ and $k_{s}$ are the density, specific heat capacity and thermal conductivity of the substrate material, $T_{s}$ is the surface temperature from TFG and tis the time. A cubic spline fitting is used to discretize the temperature signal required for recovering heat flux $(6,7)$ :

$$
\begin{aligned}
{\left[T_{S}(\tau)\right]_{C S}=} & C_{1, i}+C_{2, i}\left(\tau-\tau_{i}\right) \\
& +\frac{1}{2} C_{3, i}\left(\tau-\tau_{i}\right)^{2}+\frac{1}{6} C_{4, i}\left(\tau-\tau_{i}\right)^{3} \\
\tau_{i} \leq \tau \leq & \tau_{i-1}, \quad i=1,2, \ldots M
\end{aligned}
$$

where $\tau=S_{t} t$ is the scaled time and $S_{t}$ is the scaling factor which is considered as unity in this case. The co-efficient is calculated as follows:

$$
C_{n, i}=\frac{\mathrm{d} T_{s}^{n-1}\left(\tau_{i}\right)}{\mathrm{d} \tau}
$$

$$
\begin{aligned}
& \dot{q}_{r}\left(\tau_{M+1}\right) \\
& =\left\{\begin{array}{r}
2 \sqrt{\frac{\rho_{s} c_{s} k_{s}}{\pi}} \sum_{i=1}^{M-1} V_{i}\left(P_{i}^{\frac{1}{2}}-R_{i}^{\frac{1}{2}}\right)-\frac{W_{i}}{3}\left(P_{i}^{\frac{3}{2}}-R_{i}^{\frac{3}{2}}\right)+\frac{C_{4, i}}{10}\left(P_{i}^{\frac{5}{2}}-R_{i}^{\frac{5}{2}}\right) \\
+2 \sqrt{\frac{\rho c k}{\pi}}\left(V_{M} P_{M}^{\frac{1}{2}}-\frac{W_{M}}{3} P_{M}^{\frac{3}{2}}+\frac{C_{4, M}}{10} P_{M}^{\frac{5}{2}}\right)
\end{array}\right\} \sqrt{S_{t}} ; \\
& M=1,2, \ldots, J-1 \\
& \text { where } \\
& P_{i}=\tau_{M-1}-\tau_{i} ; \quad R_{i}=\tau_{M+1}-\tau_{i+1} ; \\
& F_{i}=C_{1, i}+C_{2, i} P_{i}+\frac{C_{3, i}}{2} P_{i}^{2}+\frac{C_{4, i}}{6} P_{i}^{3} ; \\
& V_{i}=\frac{\mathrm{d} F_{i}}{\mathrm{~d} \tau_{M+1}} ; \quad W_{i}=\frac{\mathrm{d}^{2} F_{i}}{\mathrm{~d} \tau_{M+1}^{2}}
\end{aligned}
$$

In order to use this equation (Eq. 8), it is desired to have closed form solution of transient temperature data from experimental signal. The polynomial equation using cubicspline method (Eq. 6) is used in the present work to discretize the temperature data for obtaining a closed form solution $[9,11]$. Using this procedure, the surface heat fluxes are estimated from the temperature histories and are 


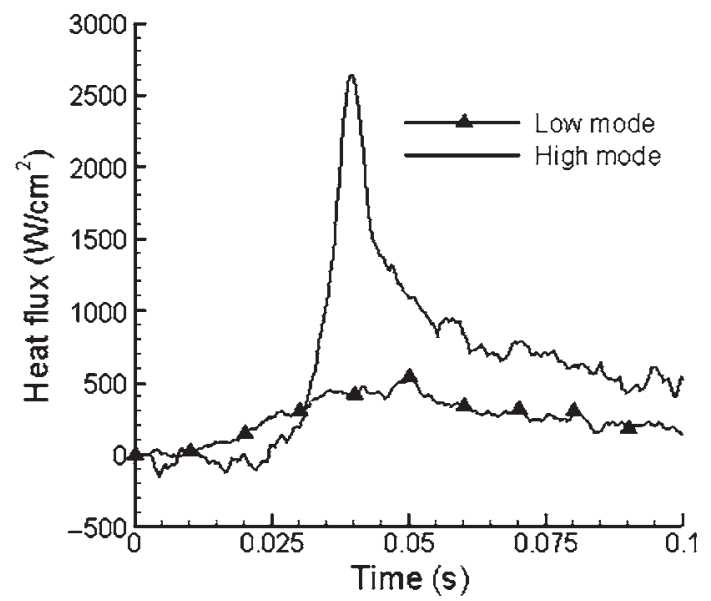

Figure 5. Surface heat history from recovered through onedimensional heat conduction modeling.

shown in figure 5. After the initial period, there is sudden rise in surface heat flux and the rate of rise is faster when the gauge experiences higher heat load. After the initial transience, the average value of step surface heat flux noticed for high mode is $\left(415 \mathrm{~W} / \mathrm{cm}^{2}\right)$ whereas for low mode is $\left(196 \mathrm{~W} / \mathrm{cm}^{2}\right)$. The surface heat fluxes deduced are shown in figure 5. In 'low mode' the rise in heat flux is gradual (i.e. 'ramp') and in other case, there is a sudden rise indicating 'step' heat load. This shows the capability of TFGs for responding changes in temperatures in both cases. However, the 'step' nature of heat load is more analogous to situations in high enthalpy facilities where the test-flow durations are $1 \mathrm{~ms}$ or less. In this short time scale, the convective heat load is typically 'step' in nature so that Eq. (5) can be used to obtain the time average values of heat flux from the transient temperatures. In this case, the values are calculated for both the mode of operations and are compared in table 2. When compared with the input heat flux values based on the nozzle area, the deviation is seen to be larger (32\%) in 'low mode' and smaller (4\%) in 'high mode'. It essentially means that the sudden rise in convective heat load in short time is recovered more accurately through this one-dimensional modeling based on semi-infinite substrate assumption. The thermal penetration of heat into the substrate becomes significant when there is gradual rise in heat flux. Thus, the accuracy of predictions of surface heat flux drops through one-dimensional heat conduction modeling with semi-infinite assumption. The other possible cause of source of discrepancy may be the unaccountable convective losses even though sufficient care has been taken during the experiments.

\subsection{Numerical simulation}

The aim of this investigation is to provide an insight about flow parameters and estimate heat flux at the senor location with prevailing experimental parameters. For this purpose,

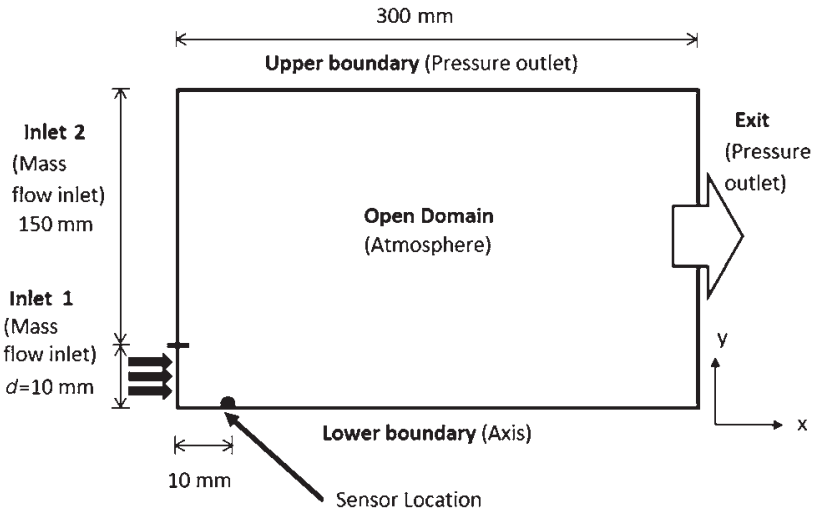

Figure 6. Computational domain for flow field simulation.

an empty half-domain with appropriate dimensions is chosen and experimental flow conditions are simulated for transient incompressible flow in an axisymmetric geometry (figure 6). Using the commercial package (ANSYS-FLUENT v.14.5), the flow is simulated as an axial jet of heated air entering to the domain. The inlet of the domain is modeled in two parts; 'inlet 1' having a mass flow inlet of $0.0018 \mathrm{~kg} / \mathrm{s}$ and 'inlet 2' with no mass flow (i.e. stagnant atmospheric inlet at temperature of $25^{\circ} \mathrm{C}$ ). The total mass flow is considered only in the horizontal direction and any vertical velocity is neglected at inlet. The mass flow rate for 'inlet 1' is same for both modes of operation of hot-air gun. But the inlet temperature is kept at $300^{\circ} \mathrm{C}$ and $500^{\circ} \mathrm{C}$ for low and high mode, respectively. The width of the 'inlet-1' is chosen based on the nozzle radius $(d=10 \mathrm{~mm})$ of the hot-air gun while the choice of width of 'inlet-2' is arbitrary keeping in view that the upper boundary is far enough to be effected by the nozzle flow (figure 6). Also, a half domain is considered since the flow is symmetrical along the horizontal axis and also to reduce computation time. Thus, the computational half-domain has a length of $300 \mathrm{~mm}$ with height of $160 \mathrm{~mm}$ while maintaining symmetry along the axis. Uniform quad meshing has been applied over the domain and the mesh size is adapted as a result of mesh independent study. The time step size had been fixed at $1 \mathrm{~ms}$ with the number of steps as 100 . The convergence criterion had been fixed at $10^{-6}$ with number of iterations as 50 per step. In order to validate the simulation study in terms of accuracy, the nondimensional velocity profiles $\left(u / u_{c}\right)$ are plotted (figure $7 \mathrm{a}$ ) as a function of non-dimensional distance $\left(y / y_{d}\right)$ at different axial locations $(x / d)$. It replicates classical nature of self-similar velocity profiles with $\left(y_{d}\right)$ as the half width of the jet - the distance from the jet axis where velocity is half the axial velocity $\left(u_{c}\right)$. The present computation matches well with the Gaussian type structure as reported in literature [22]. Further, the absolute values of velocity vectors along axial distance are plotted in figure $7 \mathrm{~b}$. The jet velocity of $10 \mathrm{~m} / \mathrm{s}$ as modeled in 'inlet 1 ' in 'high mode' 


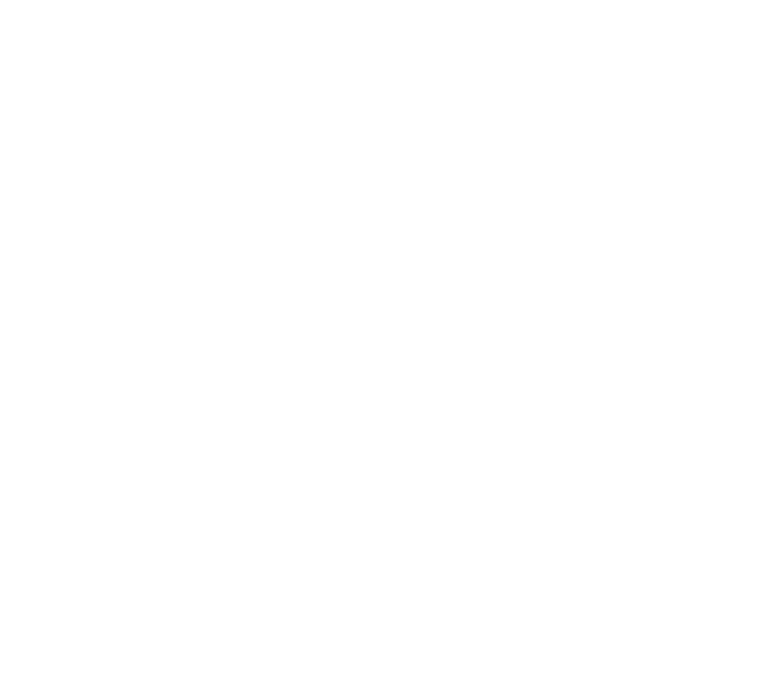

(a)

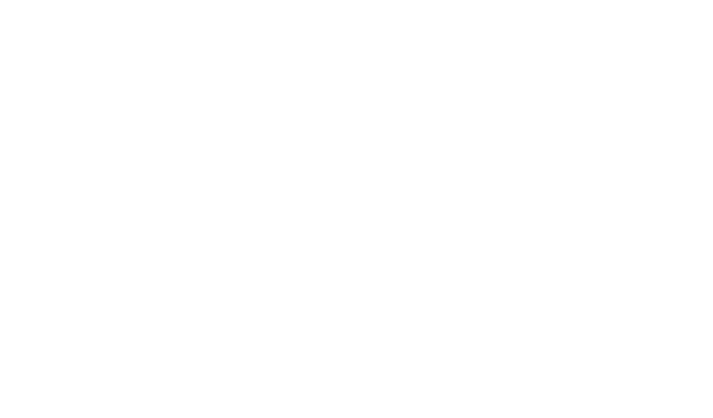

(b)

Figure 7. (a) Self-similar velocity profile; (b) velocity vectors in the computational flow field.

seems to be unaffected till $50 \mathrm{~mm}$ from the axial distance. As the axial distance increases, it interacts with the surrounding air and the velocity gradient in vertical direction becomes comparable to the axial velocity (figure $7 b$ ). However, at the location of TFG during experiment (i.e. 10 $\mathrm{mm}$ in the axial direction), no significant entrapment of atmospheric air is noticed. It further reconfirms the accuracy of the simulation for the present investigation and resembles the behavior of classical velocity profile for a hot-air jet expanding in atmosphere [22, 23]. The average heat fluxes $\left(\dot{q}_{n}\right)$ at the senor location $(10 \mathrm{~mm})$ are estimated to be $278 \mathrm{~W} / \mathrm{cm}^{2}$ and $411 \mathrm{~W} / \mathrm{cm}^{2}$, for low and high modes, respectively (table 2). The deviation in terms of predictions of surface heat flux is seen to be small (i.e. 5\%) for higher convective heat loads in short time scale. The accuracy of prediction drops when time scale of experiment increases for which there is gradual rise in convective heat load. It essentially highlights the fact of effectiveness of TFGs for inferring surface heat fluxes from transient temperatures in short duration experiments.

\section{Uncertainty analysis}

In the experimental investigation, uncertainty assessment deals with the accuracies involved in the instruments and subsequently its effects in the global measurements. The instruments used in the present investigations include thermometer, source meter and hot-air gun and constant power supply and as per manufacturers' specification, the uncertainty in these equipment's are $\pm 0.002^{\circ} \mathrm{C}, \pm 0.02 \%$ and $\pm 0.12 \%$, respectively. Sequential perturbation technique has been used to calculate the uncertainty in this experiment [24]. The uncertainty value for TCR estimation is found to be $\pm 0.25 \%$ and the uncertainties involved in temperature and heat flux are $\pm 0.3 \%$ and $\pm 0.4 \%$, respectively.

\section{Conclusion}

The performance of a STFG fabricated in-house is analysed by exposing it in a convective heating environment. The heat load resembles the nature of a 'step' in which the hot air flow prevails over the TFG for $100 \mathrm{~ms}$. The TCR value of this RTD type sensor is obtained through oil-bath calibration experiment and is found to be $0.0026 /{ }^{\circ} \mathrm{C}$. The convective experiments are performed with a hot-air gun of known wattage and voltage histories are obtained from the TFG. With the knowledge of TCR, the temperature history is obtained. The transient surface heat flux is recovered from the temperature history by using one-dimensional heat conduction modeling. Further, the numerical investigation is carried out under prevailing experimental conditions in an empty domain. The self-similarity profile of velocity verifies the accuracy of the simulation and the heat flux is computed. The results from all these investigations are compared critically and they show a good agreement with reasonable deviation of $4 \%$ in 'high mode' and $32 \%$ in 'low mode' of hot-air gun operation. This study reconfirms the ability of thin film gauges for their usages in practical purposes to recover convective heat fluxes in short duration transient experiments.

\section{References}

[1] Liu G, Han M and Hou W 2015 High-resolution and fastresponse fiber-optic temperature sensor using silicon Fabry- 
Pérot cavity. Opt. Express 23(6): 7237-7247. doi:10.1364/ OE.23.007237

[2] Lupan O, Ursaki V V, Chai G, Chow L, Emelchenko G A, Tiginyanu I M, Gruzintsev A N and Redkin A N 2010 Selective hydrogen gas nanosensor using individual $\mathrm{ZnO}$ nanowire with fast response at room temperature. Sens. Actuators B 144: 56-66

[3] Minakov A A and Schick C 2015 Dynamics of the temperature distribution in ultra-fast thin-film calorimeter sensors. Thermochim. Acta 603: 205-217

[4] Schultz D L and Jones T V 1973 Heat transfer measurements in short duration hypersonic facilities. AGARDograph-AG165

[5] Simmons J M 1995 Measurement techniques in high-enthalpy hypersonic facilities. Exp. Therm. Fluid Sci. 10: 454-469

[6] Wildmann N, Mauz M and Bange J 2013 Two fast temperature sensors for probing of the atmospheric boundary layer using small remotely piloted aircraft (RPA). Meas. Technol. 6: 2101-2113. doi:10.5194/amt-6-2101-2013

[7] Azerou B, Garnier B and Lahmar J 2012 Thin film heat flux sensors for accurate transient and unidirectional heat transfer analysis. J. Phys. Conf. Ser. 395: 012084. doi:10.1088/17426596/395/1/012084

[8] Kumar R, Sahoo N, Kulkarni V and Singh A 2011 Laser based calibration technique for thin film sensors for short duration transient measurements. ASME J. Therm. Sci. Eng. Appl. 3: 0445041-0445046

[9] Cook W J and Felderman E J 1966 Reduction of data from thin film heat transfer gauge: A concise numerical technique. AIAA J. 4: 561-562

[10] Li Y, Wang G and Chen H 2015 Simultaneously estimation for surface heat fluxes of steel slab in a reheating furnace based on DMC predictive control. Appl. Therm. Eng. 80: 396-403

[11] Taler J 1996 Theory of transient experimental techniques for surface heat transfer. Int. J. Heat Mass Transfer 39: 3733-3748

[12] Sahoo N and Peetala R K 2010 Transient temperature data analysis for a supersonic flight test. ASME J. Heat Transfer 132: $310-315$
[13] Sahoo N, Saravanan S, Jagadeesh G and Reddy K P J 2006 Simultaneous measurement of aerodynamic and heat transfer data for large angle blunt cones in hypersonic shock tunnel. Sadhana Acad. Proc. Eng. Sci. 31: 557-581

[14] Chana K S and Wilson T S 2001 High bandwidth heat transfer measurements in internal combustion engine under low load motored conditions. Heat transfer and cooling in propulsion and power system, RTO-MP-069(I)

[15] Hea S, Mencha M M and Tadigadapa S 2006 Thin film temperature sensor for real-time measurement of electrolyte temperature in a polymer electrolyte fuel cell. Sens. Actuators A Phys. 125: 170-177

[16] Smith D E, Bubb J V, Popp O, Diller T E and Hevey S J 1999 A comparison of radiation versus convection calibration of thin-film heat flux gauges. Proc. ASME Heat Transf. Div. HTD 364: 79-84

[17] Kumar R, Sahoo N and Kulkarni V 2012 Conduction based calibration of handmade platinum thin film heat transfer gauges for transient measurements. Int. J. Heat Mass Transfer 55: 2707-2713

[18] Kinnear K M and Lu F K 1997 Design, calibration and testing of transient thin film heat transfer gauges. NASA/ UTA Center for Hypersonic Research, The University of Texas at Arlington, Arlington, TX 76019: 1-16

[19] Sinclair I R 1987 Electronics for electricians and engineers. Industrial Press Inc, U.S.

[20] Kumar R 2014 Design, fabrication and novel calibration techniques for heat transfer gauges during short duration transient measurement. PhD Thesis, Indian Institute of Technology Guwahati

[21] Doebelin E O 1990 Measurement systems application and design. 4th ed., McGraw-Hill Publishing Company, Singapore

[22] Otiigen M V and Namer I 1988 Rayleigh scattering temperature measurements in a plane turbulent air jet at moderate Reynolds numbers. Exp. Fluids 6: 461-466

[23] Cziesla T, Biswas G, Chattopadhyay H and Mitra N K 2001 Large eddy simulation of flow and heat transfer in an impinging slot jet. Int. J. Heat Fluid Flow 22: 500-508

[24] Kline S J and McClintock F A 1953 Describing uncertainties in single sample experiments. Mech. Eng. 75: 3-8 